\title{
Genetic Evaluation and Physico-chemical Properties of Chillies (Capsicum annuum L.)
}

\author{
Sarujpisit Payakhapaab ${ }^{1}$, Danai Boonyakiat ${ }^{1} \&$ Maneechat Nikornpun ${ }^{1}$ \\ ${ }^{1}$ Department of Plant Science and Natural Resources, Faculty of Agriculture, Chiang Mai University, Chiang \\ Mai, Thailand \\ Correspondence: Maneechat Nikornpun, Department of Plant Science and Natural Resources, Faculty of \\ Agriculture, Chiang Mai University, Chiang Mai, Thailand. Tel: 66-5394-4040. E-mail: \\ maneechat.n@gmail.com
}

Received: August 16, 2012 Accepted: August 28, 2012 Online Published: November 15, 2012

doi:10.5539/jas.v4n12p253 URL: http://dx.doi.org/10.5539/jas.v4n12p253

\begin{abstract}
Cytoplasmic male sterility of chilli cultivars was evaluated for their prospective genotypes. Fifteen pairs of $F_{1}$ hybrids were evaluated for their male pollen viabilities in three natures. Hybrids which showed no pollen occurred in 9 varieties whose male parents should have the prospective genotypes of $\mathrm{N} r f r f$ or B line. Hybrids with fertile pollens in some plants and sterile ones on others appeared in 4 varieties whose male parents should have prospective genotypes of $\mathrm{N} / \mathrm{S} R f r f$. Hybrids showing fertile pollens were found in 2 varieties whose male parents should have the male sterility controlling genes in the nucleus as N/S RfRf or $\mathrm{C}$ line. Horticultural characteristics of six accessions of chillies were recorded. Chilli fruits of the accessions were analyzed for the physico-chemical properties in terms of moisture content, total soluble solids, vitamin C, capsaicin content and skin color. Accessions CA1445, CA1449 and CA1450 were found to have suitable characteristics as maintainer lines.
\end{abstract}

Keywords: Capsicum, chilli, male sterility, capsaicin, maintainer, physico-chemical properties

\section{Introduction}

Male sterility trait in pepper was first found in Capsicum frutescens (Martin \& Crawford, 1951). Subsequently, Peterson (1958) found male sterility caused by interaction between cytoplasm (S-type) and the genes in the nucleus ( $r f$ ) in Capsicum annuum. Shifriss (1997) studied and segregated various cases of natural sterile male pollens and concluded that male sterility was controlled by both the genes in the nucleus and the interaction between cytoplasm and the genes in the nucleus. Hybrid vegetable seeds generally produce high yield, good quality and consistent outputs (Khanobdee, 1992). However, producing hybrid seeds using hand emasculation of male pollen method is costly. The alternative method becomes the assurance of male sterility trait in hybrid seeds (Maneechat, 1999). Duvick (1959) exploited CMS for production of hybrid seeds with outstanding hybrid features to reduce cost associated with hand emasculation of male pollens of the parental lines, while assuring the hybrid seeds will be free from self-pollination action contamination (Mulyantoro et al., 2009; Wang et al., 2006). The farmers using hybrid seed chilli will be assured of consistent and higher output, especially from expressive heterosis in chilli hybrid seeds those results from heterozygosity (Bosland \& Votava, 2000; Kordus, 1991).

\section{Materials and Methods}

\subsection{Genetics Identification of Chilli by Pollen Viability}

Sixteen accessions of Capsicum annuum L. were grown at Chiang Mai University during the winter of 2010-11. Average day temperature was $31.2 \pm 0.7^{\circ} \mathrm{C}$, average night temperature was $16.0 \pm 0.9^{\circ} \mathrm{C}$, relative humidity was $67.8 \pm 2.4 \%$ and light intensity was $331.3 \mathrm{~W} / \mathrm{m}^{2}$. Thirty days old seedlings were transplanted into $8 \times 12$ inch black plastic bags containing rice hulls and sandy soil mixed in 1:1 ratio, additionally, $0.5 \mathrm{~kg}$ of cow manure and $10 \mathrm{~g}$ of fertilizer $15 \mathrm{~N}-15 \mathrm{P}-15 \mathrm{~K}$ were mixed into the mixed soil in each bag. They were grown in a greenhouse. Liquid fertilizer containing $150 \mathrm{~g}$ of $15 \mathrm{~N}-0 \mathrm{P}-0 \mathrm{~K}, 65 \mathrm{~g}$ of $13 \mathrm{~N}-0 \mathrm{P}-42 \mathrm{~K}, 75 \mathrm{~g}$ of $0 \mathrm{~N}-52 \mathrm{P}-34 \mathrm{~K}$ and $5 \mathrm{~g}$ of trace element which consisted of $\mathrm{Mg} 9.0 \%$, Fe $4.0 \%$, Mn 4.0\%, Cu 1.5\%, Co $0.05 \%$, Zn 1.5\%, B 0.5\% and Mo 0.1\% were mixed in 100 liters of water. Insecticides such as imidacloprid, fipronil sulfur and methomyl were used at recommended rates once a week. The 16 accessions were crossed onto 3 cytoplasmic male sterile chillies obtained from the Asian Vegetable Research and Development Center, Taiwan, who transferred these lines to 
Kasetsart University, Kampangsan. About 50 days after anthesis when the fruits were at red ripe stage, seeds were harvested and dried. The $\mathrm{F}_{1}$ hybrids were grown for evaluation of the viability of their pollens. A completely randomized design was used. Thirty plants were grown for each $F_{1}$ hybrid using the same place and same cultural practices as mentioned above. Pollen from open-flowers was stained with $1 \%$ acetocarmine to score for pollen fertility (Rai et al., 2001; Pakozdi et al., 2002; Yoon et al., 2006). The red stained color and morphology of pollen indicating the viability of pollen were basis for classifying different genotypes. They were classified as follows:

a. If all plants of a cross possessed non-viable pollen, then the male parent of that $\mathrm{F}_{1}$ hybrid was designated as sterile maintainer genes residing in the nucleus ( $(f r f)$, and normal cytoplasm $(\mathrm{N})$.

b. If all $F_{1}$ plants of a cross possessed viable pollen, then that male parent was designated as restorer genes residing in the nucleus as $R f R f$ genotype and the cytoplasm is either normal $(\mathrm{N})$ or sterile $(\mathrm{S})$.

c. If some $F_{1}$ plants of a cross possessed viable pollen and some non-viable pollen, then the male parent was designated as heterozygous for restorer genes in the nucleus (Rfrf), and the cytoplasm was conserved either normal $(\mathrm{N})$ or sterile $(\mathrm{S})$.

\subsection{Determination of the Horticultural Characteristics and Physico-chemical Properties of Chilli}

Long green chilli varieties : CA683, CA1445, CA1447, CA1448, CA 1449 and CA1450 were selected from the germplasm and compared with three control varieties. A randomized block design with three replications was used. Ten plants were used in each treatment. They were planted in a double row bed, at a spacing of $50 \times 50 \mathrm{~cm}$ with a plot size of $4.5 \mathrm{~m}^{2}$ and using the same cultural practices as mentioned above. Horticultural characteristics were also recorded (IPGRI, AVRDC, \& CATIE, 1995). The green mature fruit was analyzed for the physico-chemical properties; total soluble solids, moisture content, vitamin C (Ranganna, 1986). The capsaicin content was measured as mentioned using spectrophotometry at $750 \mathrm{~nm}$ (Anan et al., 1996). The fruit color was measured using a chromometer.

\section{Results}

Fifteen $F_{1}$ hybrids were evaluated. They were classified into three groups (Table 1) according to their pollen viability (Figure 1). Nine $F_{1}$ hybrids showed no pollen fertility. This group consisted of : PEPAC 32-84 $\times$ CA1286-1, PEPAC 32-80 × CA1286-4, PEPAC 32-92 × CA1303-6, PEPAC 32-94 × CA1303-8, PEPAC 32 -103 $\times$ CA1441-J-8, PEPAC 38-25 × CA1442-5, PEPAC 38-11 × CA1445-1, PEPAC 32-37 × CA1449-3-9 and PEPAC 36-17 × CA1450-7. Accordingly, the male parental accessions: CA1286-1, CA1286-4, CA1303-6, CA1303-8, CA1441-J-8, CA1442-5, CA1445-1, CA1449-3-9 and CA1450-7 should have prospective genotypes of $\mathrm{N} r f r f$. Four $\mathrm{F}_{1}$ hybrids showed no pollen or sterile pollen on some plants and fertile pollens on other plants in the same hybrid. This group consisted of: PEPAC 38-18 $\times$ CA1303-2, PEPAC 32-110 $\times$ CA1443-5, PEPAC 32-23 $\times$ CA1448-1-3 and PEPAC 32-7 $\times$ CA1448-5-13. Accordingly, the male parental accessions: CA1303-2, CA1443-5, CA1448-1-3 and CA1448-5-13 should have prospective genotypes of N/S Rfrf. Two $\mathrm{F}_{1}$ hybrids showed fertile pollens. This group consisted of: PEPAC 38-7 × CA683-3 and PEPAC 32-18 $\times$ CA1447-4-12. Accordingly, the male parental accessions: CA683-3 and CA1447-4-12 should have prospective genotypes of N/S RfRf.

Horticultural characteristics (IPGRI, AVRDC, \& CATIE, 1995) were recorded for 6 selected varieties: CA683, CA1445, CA1447, CA1448, CA1449 and CA1450 and three control varieties: Jakkrapat, JomThong 2 and YokSiam as presented in Table 2. Plant and fruit characteristics of some varieties as shown in figure 2-4. The fruit of the hot chilli varieties CA 1447, CA1448 and CA1450 had a higher moisture content than those of other varieties and all control varieties (Table 3 ). The results indicated among the varieties tested, the moisture content, total soluble solids (TSS), vitamin C, capsaicin content and fruit color values differed at statistically significant levels. Total soluble solid values range between 4.30-6.93\% with varieties CA1447, CA1448 and CA1450 having the highest values when compared with the others. The vitamin $\mathrm{C}$ content was expressed on the fresh weight basis of the chillies. The varieties CA1447, CA1448, CA1450, JomThong 2 and YokSiam had the highest vitamin C content. The group of varieties CA683 and CA1449 had the highest capsaicin when compared with other varieties. Canopy widths of all varieties show no statistical difference (Table 4). 
Table 1. Prospective genotype of the $\mathrm{F}_{1}$ hybrid and the male parents of chilli

\begin{tabular}{|c|c|c|}
\hline \multirow{2}{*}{$\mathrm{F}_{1}$ hybrid } & \multicolumn{2}{|c|}{ Prospective genotype } \\
\hline & $\mathrm{F}_{1}$ hybrid & Male parent \\
\hline PEPAC 32-84 × CA1286-1 & $\mathrm{S} r f r f$ & $\mathrm{~N} r f r f$ \\
\hline PEPAC 32-80 × CA1286-4 & S rfrf & $\mathrm{N} r f r f$ \\
\hline PEPAC 32-92 × CA1303-6 & S rfrf & $\mathrm{N} r f r f$ \\
\hline PEPAC 32-94 × CA1303-8 & S r rfrf & $\mathrm{N} r f r f$ \\
\hline PEPAC 32-103 × CA1441-J-8 & S r rfrf & $\mathrm{N} r f r f$ \\
\hline PEPAC 38-25 × CA1442-5 & S rfrf & $\mathrm{N} r f r f$ \\
\hline PEPAC 38-11 × CA1445-1 & S rfrf & $\mathrm{N} r f r f$ \\
\hline PEPAC 32-37 × CA1449-3-9 & S rfrf & $\mathrm{N} r f r f$ \\
\hline PEPAC 36-17 × CA1450-7 & S rrfrf & $\mathrm{N} r f r f$ \\
\hline PEPAC $38-18 \times$ CA1303-2 & S Rfrf: S rfrf & N/S Rfrf \\
\hline PEPAC 32-110 × CA1443-5 & S Rfrf: S rfrf & N/S Rfrf \\
\hline PEPAC 32-23 × CA1448-1-3 & S Rfrf: S rfrf & N/S Rfrf \\
\hline PEPAC 32-7 × CA1448-5-13 & S Rfrf: S rfrf & N/S Rfrf \\
\hline PEPAC 38-7 × CA683-3 & S Rfrf & $\mathrm{N} / \mathrm{S} R f R f$ \\
\hline PEPAC $32-18 \times$ CA1447-4-12 & $\mathrm{S} R f r f$ & $\mathrm{~N} / \mathrm{S} R f R f$ \\
\hline
\end{tabular}

Note: $\mathrm{N} r f r f$-normal cytoplasm and homozygous recessive of restorer genes which designated as maintainer, $\mathrm{N} / \mathrm{S}$ Rfrf- normal or sterile cytoplasm and heterozygous of restorer genes which designated as restorer and N/S Rfrfnormal or sterile cytoplasm and homozygous dominant of restorer genes which designated as restorer.

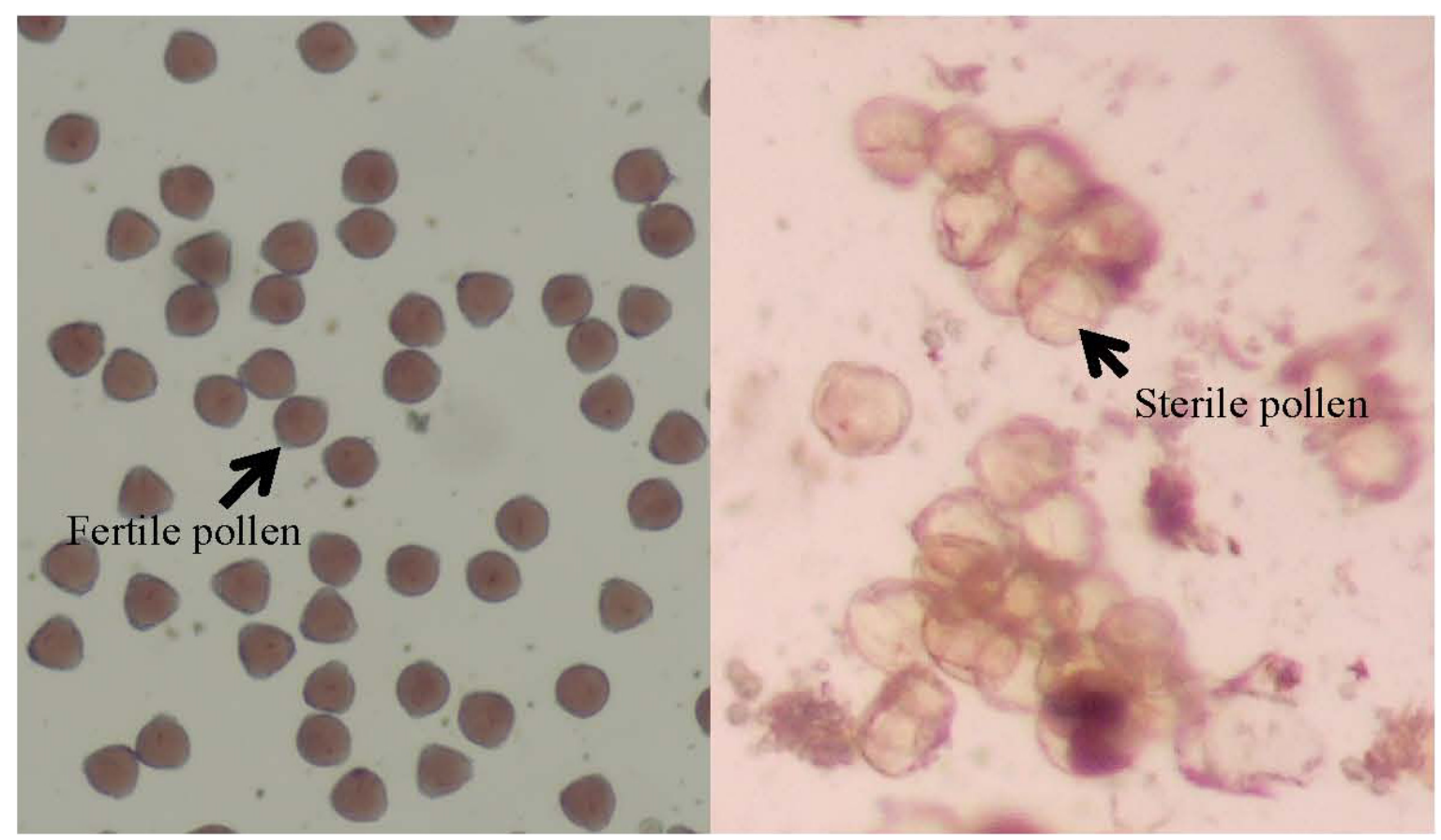

Figure 1. Fertile and sterile pollen of chilli accessions 
Table 2. Vine, inflorescence and fruit characteristics and vine growth habit of chillies

\begin{tabular}{|c|c|c|c|}
\hline Descriptors for Capsicum (1995) & CA683 & CA1445 & CA1447 \\
\hline \multicolumn{4}{|l|}{ 1. Plant descriptors } \\
\hline 1.1 Stem color & 1(Green) & 1(Green) & 1(Green) \\
\hline 1.2 Nodal anthocyanin & 5(Purple) & 5(Purple) & 5(Purple) \\
\hline 1.3 Stem shape & 1(Cylindrical) & 1(Cylindrical) & 1(Cylindrical) \\
\hline 1.4 Stem pubescence & 3(Sparse) & 3(Sparse) & 3(Sparse) \\
\hline 1.5 Plant growth habit & 7 (Erect) & 7 (Erect) & 5 (Compact) \\
\hline 1.6 Branching habit & 3(Sparse) & 5(Intermediate) & 3(Sparse) \\
\hline 1.7 Leaf density & 7(Dense) & 5 (Intermediate) & 5(Intermediate) \\
\hline 1.8 Leaf color & 3(Green) & 3(Green) & 3(Green) \\
\hline 1.9 Leaf shape & 3(Lanceolate) & 3(Lanceolate) & 3(Lanceolate) \\
\hline 1.10 Lamina margin & 1(Entire) & 1(Entire) & 1(Entire) \\
\hline 1.11 Leaf pubescence & 3(Sparse) & 3(Sparse) & 3 (Sparse) \\
\hline 1.12 Plant height $(\mathrm{cm})$ & $80.89 \pm 6.74$ & $69.33 \pm 2.40$ & $62.11 \pm 5.40$ \\
\hline 1.13 Plant canopy width $(\mathrm{cm})$ & $72.22 \pm 7.19$ & $79.78 \pm 5.09$ & $76.11 \pm 7.24$ \\
\hline 1.14 Stem length $(\mathrm{cm})$ & $27.11 \pm 2.27$ & $23.78 \pm 1.02$ & $24.89 \pm 0.19$ \\
\hline 1.15 Mature leaf length $(\mathrm{cm})$ & $20.52 \pm 3.62$ & $14.83 \pm 1.56$ & $18.26 \pm 1.18$ \\
\hline 1.16 Mature leaf width $(\mathrm{cm})$ & $5.36 \pm 0.95$ & $5.40 \pm 0.40$ & $6.87 \pm 0.59$ \\
\hline \multicolumn{4}{|l|}{ 2. Inflorescence descriptors } \\
\hline 2.1 Days to flowering & 72 & 71 & 69 \\
\hline 2.2 Flower position & 3(Pendant) & 3(Pendant) & 3(Pendant) \\
\hline 2.3 Corolla color & 1(White) & 1(White) & 1(White) \\
\hline 2.4 Corolla spot color & no & no & no \\
\hline 2.5 Corolla shape & 1(Rotate) & 1(Rotate) & 1(Rotate) \\
\hline 2.6 Anther color & 5(Purple) & 5(Purple) & 5(Purple) \\
\hline 2.7 Filament color & 1(White) & 1(White) & 6(Purple) \\
\hline 2.8 Male sterility & 0 (Absent) & 0 (Absent) & 0 (Absent) \\
\hline 2.9 Calyx pigmentation & 0 (Absent) & 0 (Absent) & 0 (Absent) \\
\hline 2.10 Calyx margin & 2(Intermediate) & 2(Intermediate) & 2(Intermediate) \\
\hline 2.11 Calyx annular constriction & 0 (Absent) & 1(Present) & 1(Present) \\
\hline \multicolumn{4}{|l|}{ 3. Fruit descriptors } \\
\hline 3.1 Anthocyanin spots or strips & 0 (Absent) & 0 (Absent) & 0 (Absent) \\
\hline 3.2 Fruit color at intermediate stage & 3 (Green) & 3(Green) & $3($ Green $)$ \\
\hline 3.3 Fruit set & 7(High) & 7(High) & 5(Intermediate) \\
\hline 3.4 Fruit color at mature stage & $8(\mathrm{Red})$ & 8(Red) & 8 (Red) \\
\hline 3.5 Fruit shape & 1(Elongate) & 1(Elongate) & 1(Elongate) \\
\hline 3.6 Fruit shape at pedicel attachment & 2(Obtuse) & 3(Truncate) & 3 (Truncate) \\
\hline 3.7 Neck at base of fruit & 0 (Absent) & 1(Present) & 1(Present) \\
\hline 3.8 Fruit shape at blossom end & 1(Pointed) & 1(Pointed) & 1(Pointed) \\
\hline 3.9 Fruit blossom end appendage & 0 (Absent) & $0($ Absent $)$ & $0($ Absent $)$ \\
\hline 3.10 Fruit cross-sectional corrugation & 3(Slightly corrugated) & 3(Slightly corrugated) & 5 (Intermediate) \\
\hline 3.11 Fruit surface & 1(Smooth) & 2(Semiwrinkled) & 2(Semiwrinkled) \\
\hline 3.12 Placenta length & $3(>1 / 2$ fruit length) & $3(>1 / 2$ fruit length) & $3(>1 / 2$ fruit length $)$ \\
\hline 3.13 Number of locules & $2-3$ & $2-3$ & $2-3$ \\
\hline 3.14 Fruit length $(\mathrm{cm})$ & $14.20 \pm 0.30$ & $13.65 \pm 0.49$ & $18.92 \pm 0.28$ \\
\hline 3.15 Fruit width $(\mathrm{cm})$ & $2.00 \pm 0.08$ & $2.12 \pm 0.06$ & $3.16 \pm 0.10$ \\
\hline 3.16 Fruit pedicel length $(\mathrm{cm})$ & $5.92 \pm 0.04$ & $4.01 \pm 0.24$ & $5.40 \pm 0.10$ \\
\hline 3.17 Fruit wall thickness (mm) & $0.193 \pm 0.01$ & $0.207 \pm 0.01$ & $0.263 \pm 0.01$ \\
\hline 3.18 Fruit weight $(\mathrm{g})$ & $16.533 \pm 2.70$ & $17.933 \pm 0.51$ & $47.500 \pm 3.35$ \\
\hline \multicolumn{4}{|l|}{ 4. Seed descriptors } \\
\hline 4.1 Seed color & 1 (Straw) & 1(Straw) & 1 (Straw) \\
\hline 4.2 Seed surface & 1(Smooth) & 1(Smooth) & 1(Smooth) \\
\hline 4.31000 -seed weight $(\mathrm{g})$ & 6.662 & 4.763 & 6.089 \\
\hline 4.4 Number of seed per fruit & $3(>50)$ & $3(>50)$ & $3(>50)$ \\
\hline
\end{tabular}


Table 2. Vine, inflorescence and fruit characteristics and vine growth habit of chillies (continue)

\begin{tabular}{|c|c|c|c|}
\hline Descriptors for Capsicum (1995) & CA1448 & CA1449 & CA1450 \\
\hline \multicolumn{4}{|l|}{ 1. Plant descriptors } \\
\hline 1.1 Stem color & 1(Green) & 1(Green) & 1(Green) \\
\hline 1.2 Nodal anthocyanin & 5(Purple) & 1(Green) & 1(Green) \\
\hline 1.3 Stem shape & 1(Cylindrical) & 1(Cylindrical) & 1(Cylindrical) \\
\hline 1.4 Stem pubescence & 3(Sparse) & 3(Sparse) & 3(Sparse) \\
\hline 1.5 Plant growth habit & 5 (Compact) & 5(Compact) & 3(Prostrate) \\
\hline 1.6 Branching habit & 5 (Intermediate) & 3(Sparse) & 5 (Intermediate) \\
\hline 1.7 Leaf density & 5(Intermediate) & 5 (Intermediate) & 5(Intermediate) \\
\hline 1.8 Leaf color & 3(Green) & 3(Green) & 3(Green) \\
\hline 1.9 Leaf shape & 3(Lanceolate) & 3(Lanceolate) & 3(Lanceolate) \\
\hline 1.10 Lamina margin & 1(Entire) & 1(Entire) & 1(Entire) \\
\hline 1.11 Leaf pubescence & 3(Sparse) & 3(Sparse) & 3(Sparse) \\
\hline 1.12 Plant height $(\mathrm{cm})$ & $58.56 \pm 2.22$ & $72.45 \pm 8.28$ & $52.89 \pm 1.02$ \\
\hline 1.13 Plant canopy width $(\mathrm{cm})$ & $66.89 \pm 1.02$ & $74.33 \pm 8.45$ & $76.22 \pm 7.07$ \\
\hline 1.14 Stem length $(\mathrm{cm})$ & $23.11 \pm 1.35$ & $19.33 \pm 1.86$ & $21.33 \pm 1.45$ \\
\hline 1.15 Mature leaf length $(\mathrm{cm})$ & $16.97 \pm 1.52$ & $17.48 \pm 1.49$ & $13.86 \pm 3.50$ \\
\hline 1.16 Mature leaf width $(\mathrm{cm})$ & $6.21 \pm 0.66$ & $6.48 \pm 1.10$ & $5.48 \pm 1.13$ \\
\hline \multicolumn{4}{|l|}{ 2. Inflorescence descriptors } \\
\hline 2.1 Days to flowering & 69 & 72 & 69 \\
\hline 2.2 Flower position & 3(Pendant) & 3(Pendant) & 3(Pendant) \\
\hline 2.3 Corolla color & 1(White) & 1(White) & 1(White) \\
\hline 2.4 Corolla spot color & no & no & no \\
\hline 2.5 Corolla shape & 1(Rotate) & 1(Rotate) & 1(Rotate) \\
\hline 2.6 Anther color & 5(Purple) & 5(Purple) & 5(Purple) \\
\hline 2.7 Filament color & 1(White) & 1(White) & 6(Purple) \\
\hline 2.8 Male sterility & 0 (Absent) & 0 (Absent) & 0 (Absent) \\
\hline 2.9 Calyx pigmentation & 0 (Absent) & 0 (Absent) & 0 (Absent) \\
\hline 2.10 Calyx margin & 2(Intermediate) & 2(Intermediate) & 2(Intermediate) \\
\hline 2.11 Calyx annular constriction & 1(Present) & 1(Present) & 1(Present) \\
\hline \multicolumn{4}{|l|}{ 3. Fruit descriptors } \\
\hline 3.1 Anthocyanin spots or strips & 0 (Absent) & 0 (Absent) & 0 (Absent) \\
\hline 3.2 Fruit color at intermediate stage & 3(Green) & 3(Green) & 3(Green) \\
\hline 3.3 Fruit set & 5(Intermediate) & 7(High) & 5 (Intermediate) \\
\hline 3.4 Fruit color at mature stage & 8(Red) & 8(Red) & 8(Red) \\
\hline 3.5 Fruit shape & 1(Elongate) & 1(Elongate) & 1(Elongate) \\
\hline 3.6 Fruit shape at pedicel attachment & Obtuse & Truncate & Truncate \\
\hline 3.7 Neck at base of fruit & 0 (Absent) & 1(Present) & 1(Present) \\
\hline 3.8 Fruit shape at blossom end & Pointed & Blunt & Pointed \\
\hline 3.9 Fruit blossom end appendage & 0 (Absent) & 0 (Absent) & 0 (Absent) \\
\hline 3.10 Fruit cross-sectional corrugation & 3(Slightly corrugated) & 5 (Intermediate) & 3(Slightly corrugated) \\
\hline 3.11 Fruit surface & 2(Semiwrinkled) & 2(Semiwrinkled) & 2(Semiwrinkled) \\
\hline 3.12 Placenta length & $3(>1 / 2$ fruit length $)$ & $3(>1 / 2$ fruit length $)$ & $3(>1 / 2$ fruit length $)$ \\
\hline 3.13 Number of locules & $2-3$ & $2-3$ & $2-3$ \\
\hline 3.14 Fruit length $(\mathrm{cm})$ & $19.26 \pm 0.67$ & $13.86 \pm 0.84$ & $15.38 \pm 0.71$ \\
\hline 3.15 Fruit width $(\mathrm{cm})$ & $3.20 \pm 0.10$ & $3.05 \pm 0.15$ & $3.26 \pm 0.16$ \\
\hline 3.16 Fruit pedicel length $(\mathrm{cm})$ & $4.99 \pm 0.20$ & $4.60 \pm 0.12$ & $5.34 \pm 0.64$ \\
\hline 3.17 Fruit wall thickness (mm) & $0.280 \pm 0.02$ & $0.247 \pm 0.01$ & $0.287 \pm 0.02$ \\
\hline 3.18 Fruit weight $(\mathrm{g})$ & $46.767 \pm 2.10$ & $32.233 \pm 1.58$ & $44.333 \pm 0.25$ \\
\hline \multicolumn{4}{|l|}{ 4. Seed descriptors } \\
\hline 4.1 Seed color & Straw & Straw & Straw \\
\hline 4.2 Seed surface & Smooth & Smooth & Smooth \\
\hline 4.3 1000-seed weight (g) & 5.033 & 7.224 & 5.477 \\
\hline 4.4 Number of seed per fruit & $>50$ & $>50$ & $>50$ \\
\hline
\end{tabular}


Table 3. Analysis of physico-chemical properties of chillies

\begin{tabular}{lcccc}
\hline Variety & Moisture (\%) & TSS (\%) & Vitamin C (mg/100 g fw.) & Capsaicin (Scoville unit) \\
\hline CA683 & $84.803 \mathrm{e}^{1}$ & $4.533 \mathrm{~d}$ & $3.846 \mathrm{c}$ & $3530.0 \mathrm{a}$ \\
CA1445 & $83.497 \mathrm{f}$ & $6.133 \mathrm{~b}$ & $5.128 \mathrm{~b}$ & $260.0 \mathrm{f}$ \\
CA1447 & $89.367 \mathrm{a}$ & $6.933 \mathrm{a}$ & $6.410 \mathrm{a}$ & $1700.0 \mathrm{~d}$ \\
CA1448 & $89.017 \mathrm{ab}$ & $6.233 \mathrm{~b}$ & $6.410 \mathrm{a}$ & $190.0 \mathrm{f}$ \\
CA1449 & $86.583 \mathrm{~d}$ & $5.167 \mathrm{c}$ & $5.128 \mathrm{~b}$ & $2750.0 \mathrm{~b}$ \\
CA1450 & $88.953 \mathrm{ab}$ & $4.567 \mathrm{~d}$ & $6.410 \mathrm{a}$ & $2370.0 \mathrm{c}$ \\
Jakkrapat (control 1) & $87.897 \mathrm{bc}$ & $4.733 \mathrm{~cd}$ & $5.128 \mathrm{~b}$ & $2610.0 \mathrm{bc}$ \\
JomThong 2 (control 2) & $87.477 \mathrm{~cd}$ & $4.833 \mathrm{~cd}$ & $6.410 \mathrm{a}$ & $960.0 \mathrm{e}$ \\
YokSiam (control 3) & $87.577 \mathrm{~cd}$ & $4.300 \mathrm{~d}$ & $6.410 \mathrm{a}$ & $1700.0 \mathrm{~d}$ \\
\hline
\end{tabular}

${ }_{1}^{1}$ Means within column with different letters differ significantly at $\mathrm{P} \leq 0.05$ according to DMRT.

Table 3. Analysis of physico-chemical properties of chillies (continue)

\begin{tabular}{llll}
\hline Variety & $\mathrm{L}^{*}$ & Chroma & Hue angle $\left(^{\circ}\right)$ \\
\hline CA683 & $48.047 \mathrm{e}^{1}$ & $42.750 \mathrm{c}$ & $130.41 \mathrm{a}$ \\
CA1445 & $53.827 \mathrm{bc}$ & $49.480 \mathrm{ab}$ & $125.99 \mathrm{~b}$ \\
CA1447 & $53.537 \mathrm{c}$ & $48.263 \mathrm{ab}$ & $125.49 \mathrm{~b}$ \\
CA1448 & $51.683 \mathrm{~cd}$ & $46.297 \mathrm{~b}$ & $126.40 \mathrm{~b}$ \\
CA1449 & $58.820 \mathrm{a}$ & $46.437 \mathrm{~b}$ & $122.65 \mathrm{c}$ \\
CA1450 & $49.987 \mathrm{de}$ & $42.927 \mathrm{c}$ & $127.13 \mathrm{~b}$ \\
Jakkrapat (control 1) & $47.750 \mathrm{e}$ & $42.153 \mathrm{c}$ & $129.60 \mathrm{a}$ \\
JomThong 2 (control 2) & $44.620 \mathrm{f}$ & $40.303 \mathrm{c}$ & $130.70 \mathrm{a}$ \\
YokSiam (control 3) & $56.830 \mathrm{ab}$ & $50.713 \mathrm{a}$ & $126.09 \mathrm{~b}$ \\
\hline
\end{tabular}

${ }^{1}$ Means within column with different letters differ significantly at $\mathrm{P} \leq 0.05$ according to DMRT.

Table 4. Canopy height and width of chillies

\begin{tabular}{lll}
\hline Variety & Height $(\mathrm{cm})$ & Width $(\mathrm{cm})$ \\
\hline CA683 & $80.89 \mathrm{a}^{1}$ & $72.22 \mathrm{~ns}$ \\
CA1445 & $69.33 \mathrm{bc}$ & 79.78 \\
CA1447 & $62.11 \mathrm{~cd}$ & 76.11 \\
CA1448 & $58.56 \mathrm{~d}$ & 66.89 \\
CA1449 & $72.45 \mathrm{ab}$ & 74.33 \\
CA1450 & $52.89 \mathrm{~d}$ & 76.22 \\
Jakkrapat (control 1) & $76.00 \mathrm{ab}$ & 73.67 \\
JomThong 2 (control 2) & $81.78 \mathrm{a}$ & 78.78 \\
YokSiam (control 3) & $74.22 \mathrm{ab}$ & 76.22 \\
\hline
\end{tabular}

${ }^{1}$ Means within column with different letters differ significantly at $\mathrm{P} \leq 0.05$ according to DMRT. 


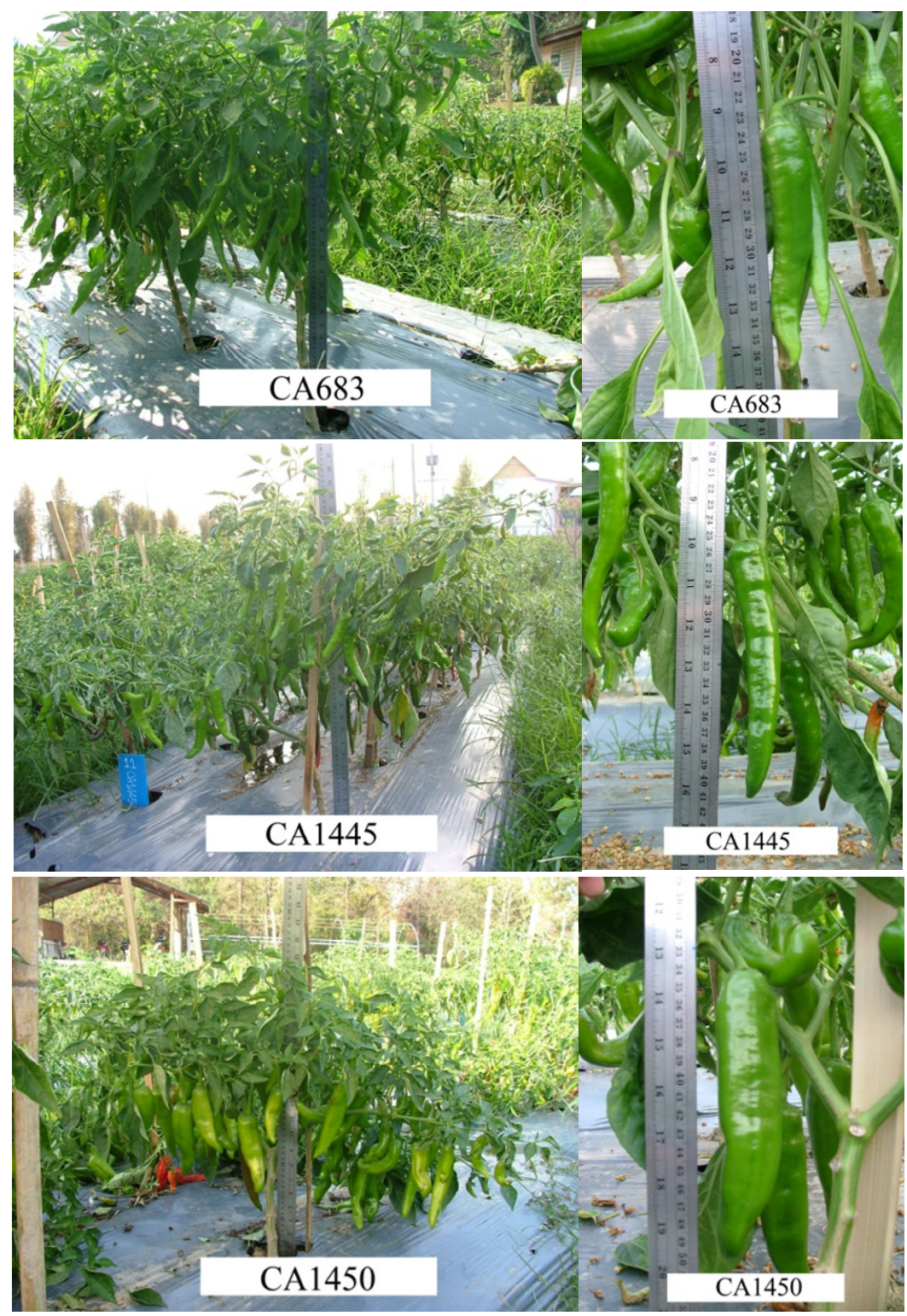

Figure 2. Plant canopy and fruit characteristics of chillies

(a) Variety CA683 (b) Variety CA1445 and (c) Variety CA1450.

\section{Discussion and Conclusion}

Male sterility is very critical for plant breeding improvement program especially for the production of hybrid seeds of chillies (Min et al., 2009) as this trait can help save time and labor, hence the production cost, as well as enhance the purity of hybrid seeds (Mulyantoro et al., 2009; Yang et al., 2008). Male sterility can be distinguished into three groups : normal male fertile chilli (N/S RfRf), male sterile chilli; ( $r f r f)$ and male fertile chilli which has heterozygosis in the genotype of the fertility restoration (N/S Rfrf) using fertility scoring for the evaluation of male sterility (Rai et al., 2001; Pakozdi et al., 2002; Yoon et al., 2006). Normal male fertile chilli (N/S RfRf) can be used as C line in breeding hybrid chillies as the parent has the homozygous male sterility controlling gene in the nucleus. This occurred in two varieties CA683 which has high capsaicin content and CA1447 which contain high moisture, total soluble solids and vitamin $\mathrm{C}$ and they can be utilized for breeding hybrids with the desirable characteristics. Nine varieties with pollen fertility were obtained. The CA1450 has high moisture content, total soluble solids and vitamin C and CA1449 contains high capsaicin and they should be exploited as maintainer or B line in breeding hybrids with these characteristics. The chilli varieties with heterozygous male sterility controlling gene may be used as $\mathrm{C}$ line in future breed improvement whose accession CA1448 has high content of moisture, total soluble solids and, vitamin C. The distribution of the $\mathrm{F}_{1}$ hybrids in the three male pollen viability groups is supported by the research results of Shifriss (1997) which enable an 
understanding of the genetic mechanism in fertility restoration and the use of male sterility trait in hybrid development (Wang et al., 2006). It is also found that the fertility scoring method is robust in genetic evaluation for the present purpose (Pakozdi et al., 2002).

\section{Acknowledgements}

The authors wish to thank the Tropical Research and Development of Vegetables Center, Kasetsart University, Kampangsan for providing hot chilli genetic sources and the Graduate School, Chiang Mai University for funding support for this dissertation research.

\section{References}

Anan, T., Ito, H., Matsunaga, H., \& Monma, S. (1996). A simple method for determining the degree of pungency of pepper. Capsicum and Eggplant Newsletter, 15, 51-54.

Bosland, P. W., \& Votava, E. J. (2000). Peppers: Vegetable and Spice Capsicums. Wallingford: CABI Publishing.

Duvick, D. N. (1959). The use of cytoplasmic male-sterility in hybrid seed production. Economic Botany, 13, 167-195. http://dx.doi.org/10.1007/BF02860581

IPGRI, AVRDC, \& CATIE. (1995). Descriptors for Capsicum (Capsicum spp.). International Plant Genetic Resources Institute, Rome, Italy; the Asian Vegetable Research and Development Center, Taipei, Taiwan, and the Centro Agronómico Tropical de Investigación y Enseñanza, Turrialba, Costa Rica.

Khanobdee, J. (1992). Vegetable Seed Production (p.183). Odeon Store, Bangkok (in Thai)

Kordus, R. (1991). Diallel analysis of some characters in pepper. Folia Horticulturae, 3(2), 51-63.

Maneechat, N. (1999). Hybrid Vegetable Seed Production (p. 132). Odeon Store, Bangkok (in Thai)

Martin, J., \& Grawford, J. H. (1951). Several type of sterility in Capsicum frutescens. Journal of the American Society for Horticultural Science, 57, 335-338.

Min, W. K., Kim, S., Sung, S. K., Kim, B. D., \& Lee, S. (2009). Allelic discrimination of the Restorer-of-fertility gene and its inheritance in peppers (Capsicum annuum L.). Theoretical and Applied Genetics, 119(7), 1289-1299. http://dx.doi.org/10.1007/s00122-009-1134-y

Mulyantoro, Chen, S. Y., Wahyono, A., \& Ku, H. M. (2009). Modified complementation test of male sterility mutants in pepper (Capsicum annuum L.): preliminary study to convert male sterility system from GMS to CMS. Euphytica, 169(3), 353-361. http://dx.doi.org/10.1007/s10681-009-9968-6

Pakozdi, K., Taller, J., Alfoldi, Z., \& Hirata, Y. (2002). Pepper (Capsicum annuum L.) cytoplasmic male sterility. Journal of Central European Agriculture, 3(2), 149-157.

Peterson, P. A. (1958). Cytoplasmically inherited male sterility in Capsicum. American Naturalist, 92, 11-119. http://dx.doi.org/10.1086/282017

Rai, S. K., Banerjee, M. K., Kalloo, G., \& Kumar, S. (2001). Cytological mechanisms of male sterility in a nuclear-cytoplasmic line of chilli pepper (Capsicum annuum L.). Capsicum \& Eggplant Newsletter, 20, 64-67.

Ranganna, S. (1986). Handbook of analysis and quality control for fruit and vegetable products. Tata: Tata Mc Graw-hill Publishing Co. Ltd.

Shifriss, C. (1997). Male sterility in pepper (Capsicum annuum L.). Euphytica, 93, 83-88. http://dx.doi.org/10.1023/A:1002947907046

Wang, L. H., Zhang, B. X., Daubeze, A. M., Huang, S. W., Guo, J. Z., Mao, S. L., Palloix, A., \& Du, Y. C. (2006). Genetics of fertility restoration in cytoplasmic male sterile pepper. Agricultural Sciences in China, 5(3), 188-195. http://dx.doi.org/10.1016/S1671-2927(06)60037-0

Yang, C. J., Chen, B. J., Liu, J. G., \& Li, S. (2008). Study on the superiority of hybrid breeding by male sterility in hot pepper. Journal of China Capsicum, 1, 36-38.

Yoon, J. B., Yang, D. C., Do, J. W., \& Park, H. G. (2006). Overcoming two post-fertilization genetic barriers in interspecific hybridization between Capsicum annuum and C. baccatum for introgression of Anthracnose resistance. Breeding Science, 56, 31-38. http://dx.doi.org/10.1270/jsbbs.56.31 